\title{
Power Flow Algorithms for Multi-terminal VSC-HVDC with Droop Control
}

\author{
Wenyuan Wang, Student Member, IEEE, and Mike Barnes, Senior Member, IEEE
}

\begin{abstract}
This paper addresses the problem posed by complex, nonlinear controllers for power system load flows employing multi-terminal voltage source converter (VSC) HVDC systems. More realistic dc grid control strategies can thus be carefully considered in power flow analysis of ac/dc grids. Power flow methods for multi-terminal VSC-HVDC (MTDC) systems are analysed for different types of dc voltage control techniques and the weaknesses of present methods are addressed. As distributed voltage control is likely to be adopted by practical dc grids, a new generalized algorithm is proposed to solve the power flow problems with various non-linear voltage droops, and the method to incorporate this algorithm with ac power flow models is also developed. With five sets of voltage characteristics implemented, the proposed scheme is applied to a five-terminal test system and shows satisfactory performance. For a range of wind power variations and converter outages, post-contingency behaviours of the system under the five control scenarios are examined. The impact of these controls on the power flow solutions is assessed.
\end{abstract}

Index Terms-Multi-terminal, Voltage source converter (VSC) HVDC, MTDC, power flow, droop control, dc grid

\section{INTRODUCTION}

A s voltage source converter (VSC) HVDC technology rapidly evolves, both the amount and the scale of VSC HVDC projects continue to rise globally [1-4]. VSC HVDC has become the most feasible solution to the integration of remotely located large wind farms, mainly due to its small footprint and its ability to support very weak ac systems. Advantages offered by a multi-terminal topology include enhanced reliability of the dc system, flexibility of power dispatch control, reduction of intermittence of renewable energy, and efficient utilization of converters and cables. Multiple plans for dc grids have been approved or are under consideration [1, 3].

DC voltage is the essential factor that indicates the power balance and the stability of an MTDC system. Compared with a conventional ac grid of large inertia, the transient response of a capacitance-based dc grid is extremely fast. Therefore it is unrealistic to rely on telecommunications for MTDC control. Local control per terminal, at most modified by a slow central coordinating control, seems to be the favoured control option. Such dc voltage control techniques can be categorised into

Manuscript submitted for review June 19, 2013. This work was supported by National Grid and The University of Manchester.

W. Wang and M. Barnes are with the School of Electrical and Electronic Engineering, The University of Manchester, Manchester, M13 9PL, U.K. (email: wenyuan.wang@student.manchester.ac.uk; mike.barnes@manchester. ac.uk).

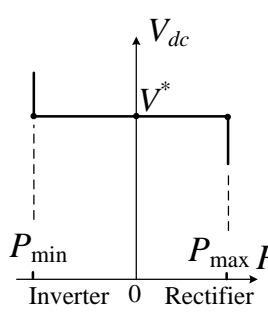

(a) Slack bus

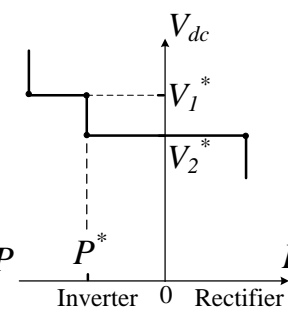

(b) Voltage margin

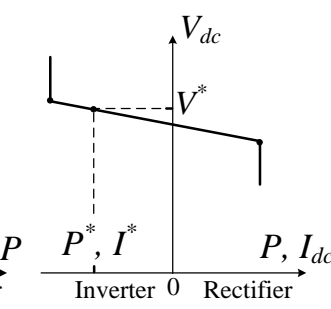

(c) Voltage droop

Fig. 1. Basic voltage characteristics for MTDC control.

centralized dc slack bus control, voltage margin control and distributed voltage droop control.

One terminal may be selected as the dc slack bus to compensate the power imbalance of the overall dc grid. However, the slack terminal may have to be over-rated and connected to a very strong ac network, and the loss of this converter may lead to the instability of the whole dc grid. Voltage margin control [5] can be considered as an improved constant dc voltage control with multiple stages of alternative slack buses. The role of voltage regulation can be taken over by another terminal once a slack bus is offline or reaches its limit. However, several disadvantages remain for voltage margin control especially in a large MTDC system.

The reliability of the dc grid can be significantly enhanced by droop control since multiple converters can simultaneously contribute to the dc voltage stability. Various types of dc voltage droop characteristics, including voltage-power (V-P) droop [6, 7], voltage-current (V-I) droop [8] and voltage droop with different dead-bands and limits [9], have been proposed for MTDC control. However, prior art has been focusing on the dynamic behaviour of voltage droop, while the steady-state aspect of various droop lines has not been addressed in detail.

Droop control is likely to be employed in the interests of MTDC dynamics and stability, however the use of such quasi-steady-state droop characteristics could significantly increase the complexity of power flows. Under such situations, the operating point and power sharing of the system vary with the power disturbances such as those imposed by wind farm power fluctuations and converter outages. As a grid supervisory control relying on telecommunications may not be available or in any case is likely to update the converter set-points only periodically in a very slow manner, the impact of the quasi-steady-state droop lines on the dc power flow needs to be well understood, especially when the standardization of the supervisory control remains unclear. Moreover, determination 


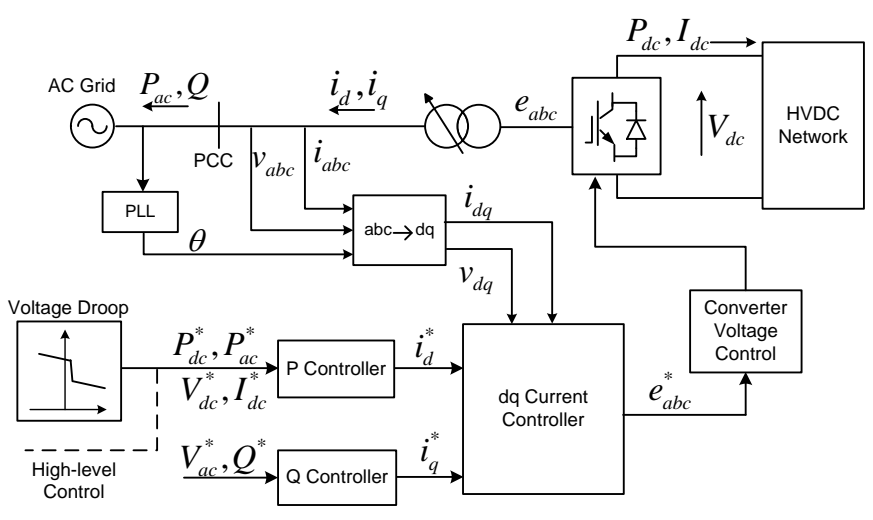

Fig. 2. Voltage droop and converter power control.

of the optimised set-points by the supervisory control could also require such power flow techniques incorporating droop characteristics. It is of great interest for grid operators to fully understand the power flow of the systems using the distributed droop control as the outermost local converter control.

Previous research has focused on solving MTDC power flow without taking detailed dc voltage control into consideration. A unified power flow method for integrated AC/MTDC systems is proposed in [10] by solving the ac and dc power flow equations simultaneously, assuming dc slack bus control. In a the ac/dc power flow algorithm discussed in [11], the ac and dc network equations are solved sequentially in each iteration. Research on power flow of dc network is provided in [7, 12-14]. Basic dc power flow analysis has been applied in [13] and [14] to represent the dc transmission losses and to optimise the voltage references. DC slack bus voltage control has been used by most papers examining power flow analysis. The sequential method is updated by including the V-P droop in [15]. V-P droop is assessed in detail using linear analysis in [7], however the non-linear power flow is not performed.

This paper aims to provide a range of power flow solutions for MTDC systems with different voltage control techniques.

\section{CONTROL OF MTDC SYSTEMS}

From the innermost to the outermost control, a cascaded MTDC control system can be structured as: converter voltage control, dq current control, real and reactive power control, voltage droop control, secondary control (optional) and supervisory control.

A simplified diagram for a VSC control system is shown in Fig. 2. The control system is a cascaded system based upon a fast and decoupled dq current control. The voltage reference for the VSC is produced by the current controller. The set-point of the current control is supplied by the real and reactive power controller, whose input may vary depending on different applications. The reference for the active power control can be generated by the voltage droop control or directly scheduled by the supervisory control via a telecommunication link.

A basic droop characteristic and the higher level grid-stage control are shown in Fig. 3, where the dashed lines indicate that communication is required. The power or current reference of the local VSC control is scheduled according to the pre-defined droop lines and measured dc voltage. The secondary control,

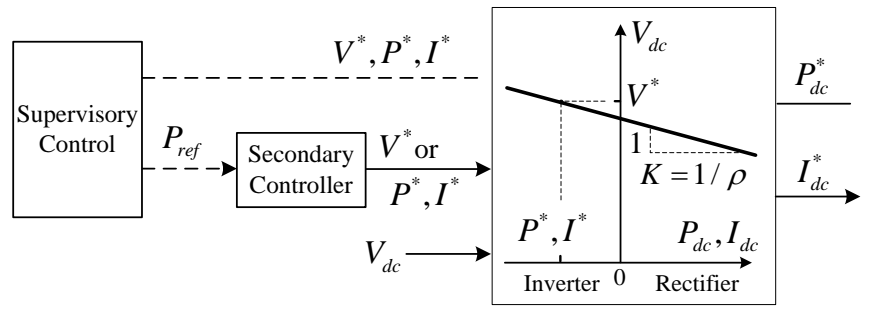

Fig. 3. Voltage droop control and outer supervisory control.

with its reference given by the supervisory control, is mainly used to adjust the droop references after contingencies in order to maintain scheduled power flow. The supervisory control is able to access and modify most control references in order to achieve an optimal operation of the dc grid. Optimal power flow (OPF) might be required for this high-level application.

Either the supervisory or the secondary control is not a necessity for the operation of the dc grid and it is likely that they may only be active periodically. Analysing the power flow resulting from the droop control is essential for understanding the quasi-steady-state behaviour of MTDC system.

\section{INTEGRATION OF AC/DC POWER FLOW}

The main novelty of this paper is the development of the dc power flow approaches which can be applied with various dc grid control designs. Since MTDC systems essentially serve ac networks, the methodology for the integration of the ac and dc power flow models is presented in this section, with the associated flow chart illustrated in Fig. 4. The ac and dc power flow are computed separately, as the power flow of the dc system is intrinsically determined by the converter dc voltage/power control setting. This approach starts by solving the dc power flow, and the computed dc side powers will then be utilized for the initialization of ac power flow. The power loss is calculated iteratively according to the results obtained from the up-to-date ac power flow, with the convergence indicated by active power at the point of common coupling (PCC) bus.

Unlike the unified ac/dc power flow algorithms, this method allows existing ac power flow models to incorporate the MTDC models with no significant modification. The key step of this algorithm is the dc system power flow with droop lines, which will be analysed in detail in Section IV-VI.

From the dc power flow point of view, the converter dc bus can be represented by a fixed or variable $\mathrm{V}_{\mathrm{dc}}$ bus or $\mathrm{P}_{\mathrm{dc}}$ bus, or more generally, the droop dc voltage characteristics. To facilitate ac power flow calculation, the PCC bus of the converter station can be described as a PV or PQ bus, depending on the reactive power control design.

A simplified power loss model is provided here to link the converter dc side power with the power injection into the ac grid at the PCC bus. According to the detailed power loss analysis for the state-of-the-art modular multi-level converter (MMC) performed in [16], the conduction loss of upper arm (UA) and lower arm (LA) can be represented by (1)-(2), where $N_{s}$ and $N_{p}$ represent the number of sub-modules in series and in parallel in each arm respectively, $V_{o}$ and $R_{o}$ denote the on-state slope voltage and resistance of IGBT/diode. 


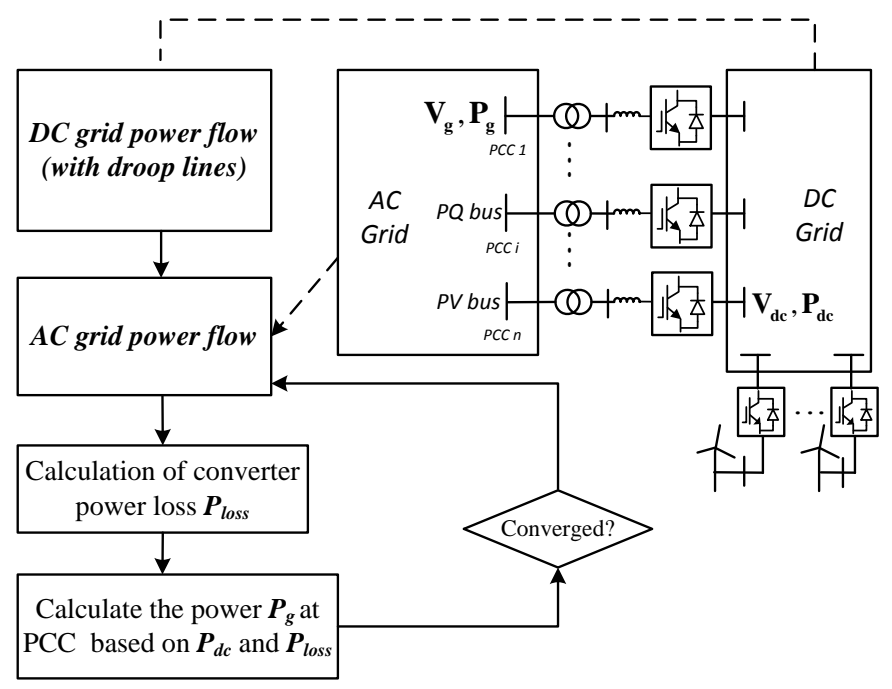

Fig. 4. Integration of MTDC power flow with ac power flow.

Assumption that the IGBT and diode have similar on-state characteristics is made to greatly simplify the power loss calculation with only a slight degradation on the accuracy with appropriate parameter choice. The total conduction loss for the converter valve can then be computed by (3).

$$
\begin{array}{r}
P_{c o n}^{U A}=V_{o} N_{s} \cdot\left|\frac{I_{a c}(\omega t)}{2}+\frac{I_{d c}}{3}\right|+\frac{R_{o} N_{s}}{N_{p}} \cdot\left[\frac{I_{a c}(\omega t)}{2}+\frac{I_{d c}}{3}\right]^{2} \\
P_{c o n}^{L A}=V_{o} N_{s} \cdot\left|\frac{I_{a c}(\omega t)}{2}-\frac{I_{d c}}{3}\right|+\frac{R_{o} N_{s}}{N_{p}} \cdot\left[\frac{I_{a c}(\omega t)}{2}-\frac{I_{d c}}{3}\right]^{2} \\
P_{c o n}=3 \cdot \int_{0}^{2 \pi}\left(P_{c o n}^{U A}+P_{c o n}^{L A}\right) d(\omega t)
\end{array}
$$

Switching loss is derived based on the general form [17]:

$$
P_{\text {switch }}=6 N_{s} N_{p} \cdot f \cdot \sum_{\text {cycle }}\left(E_{\text {on }}(I)+E_{\text {off }}(I)+E_{\text {rec }}(I)\right) \text {. }
$$

The result in [16] suggests that the switching loss only corresponds to a fraction of the total MMC valve loss. Accurate switching loss calculation requires complex non-linear modelling however this level of modelling fidelity may not be necessary for power flow calculations. The average phase current is used in (4) for simplification, as suggested in [18].

By combing (3) and (4), the total loss of the converter station can be represented as:

$$
P_{\text {loss }}=K_{1} \cdot\left|I_{a c}\right|+K_{2} \cdot\left|I_{d c}\right|+\left(K_{3}+R_{T}\right) \cdot I_{a c}^{2}+K_{4} \cdot I_{d c}^{2}
$$

where $R_{T}$ is the aggregated resistance of converter transformer and the equivalent converter reactor, and $K_{1-4}$ are constants.

\section{POWER FlOW OF MTDC WITH A Slack BuS}

Power flow analysis of an MTDC system aims to obtain the operating point of every terminal in the grid, with provided generation, loading and control conditions. The problems are usually represented by a series of non-linear relationships between voltages and currents. The power flow algorithms

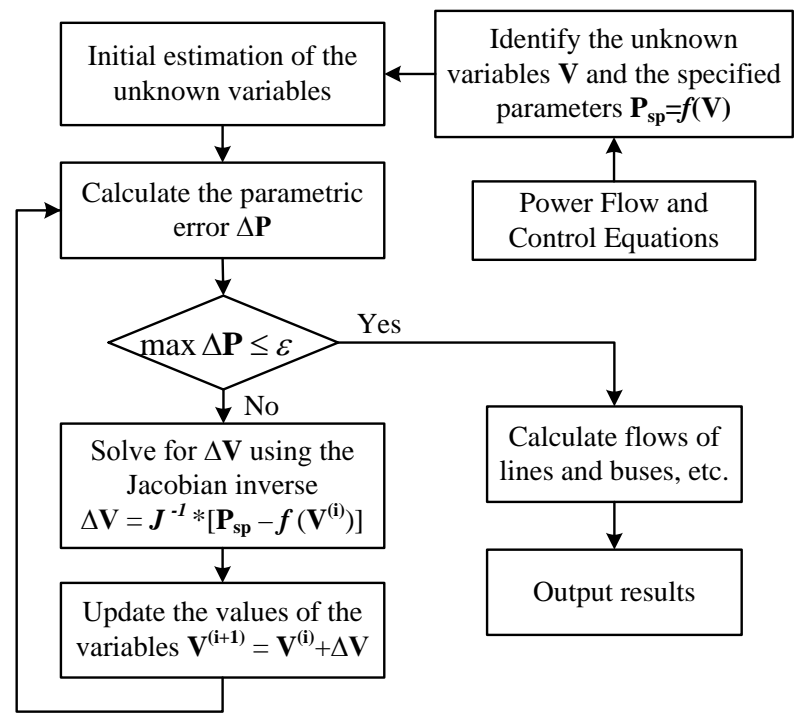

Fig. 5. Flow chart of the NR method for MTDC power flow.

proposed in this paper are based on the well-known Newton-Raphson (NR) method [19, 20].

A simplified flow chart of the numerical iterative procedure for solving a generic MTDC power flow is shown in Fig. 5. Firstly, with the system represented by the power flow and control equations, the unknown variables $\mathbf{V}$ and the specified parameters $\mathbf{P}_{\mathbf{s p}}$ need to be selected. In MTDC studies, normally dc voltages are selected as the variables and the power quantities are chosen to be the specified parameters, which can be represented using the non-linear parametric functions $f(V)$.

Based upon the existing estimates of the unknown voltages, the Jacobian matrix $\mathbf{J}$ is composed of partial derivatives of the functions $f(V)$ :

$$
\mathbf{J}=\frac{\partial \mathbf{P}_{s p}}{\partial \mathbf{V}}=\frac{\partial \mathbf{f}(\mathbf{V})}{\partial \mathbf{V}} .
$$

Based on the inverse of the Jacobian, the new set of the voltage estimates are calculated by

$$
\mathbf{V}^{(i+1)}=\mathbf{V}^{(i)}+\mathbf{J}^{-1} \cdot\left[\mathbf{P}_{s p}-\mathbf{f}\left(\mathbf{V}^{(i)}\right)\right]
$$

where $\mathbf{V}^{(i)}$ and $\mathbf{V}^{(i+1)}$ are the $i$ th and (i+1)th estimate respectively. The estimated voltages are updated iteratively until an acceptable tolerance has been achieved for the mismatch between the specified parameters and those computed using the estimated variables.

For power flow studies of various dc networks and control methodologies, the key difference is the description of the power flow equations and the resulting Jacobian matrix.

In a dc system with $n$ terminals, the steady-state relationship between the dc voltages and currents can be written as

$$
\mathbf{I}_{d c}=\mathbf{Y} \cdot \mathbf{V}_{d c}
$$

where $\mathbf{I}_{\mathbf{d c}}$ is the nodal current injection vector, $\mathbf{V}_{\mathbf{d c}}$ is the dc voltage vector and $\mathbf{Y}$ is the admittance matrix of the network.

For a symmetrical monopole HVDC system, if $\mathbf{V}_{d c}$ is comprised of pole-to-pole dc voltages, the admittance matrix needs to be calculated based on the series resistance of the 
positive-pole and negative-pole cables. Per-unit values are used for the power flow studies here as the differences between the bipolar and monopolar configurations can thus be disregarded.

As analysed by a majority of previous literature, in a basic power flow problem for a dc grid of $n$ buses, dc voltage of the slack bus is provided. The vector of the $(n-1)$ specified parameters are comprised of given nodal power injections or line branch power flows. Wind farm terminals are assumed to be power buses as normally they are not equipped with proper dc voltage control capability.

The power injected to the MTDC system by terminal $i$ can be represented as

$$
P_{i}=V_{i} \cdot\left(\sum_{j=1}^{n} Y_{i j} V_{j}\right)
$$

The branch power flow from terminal $i$ to terminal $j$ is

$$
P_{i j}=V_{i} \cdot Y_{i j}\left(V_{j}-V_{i}\right) .
$$

Accordingly, the Jacobian matrix elements associated with (9) and (10) can be derived as (11) and (12), respectively. With the $(n-1)$ non-linear equations tackled by the NR method, this basic power flow with the slack bus is solved.

$$
\begin{gathered}
\frac{\partial P_{i}}{\partial V_{i}}=2 Y_{i i} V_{i}+\sum_{j=1, j \neq i}^{n} Y_{i j} V_{j},\left.\frac{\partial P_{i}}{\partial V_{j}}\right|_{j \neq i}=V_{i} Y_{i j} \\
\frac{\partial P_{i j}}{\partial V_{i}}=Y_{i j} V_{j}-2 V_{j}, \frac{\partial P_{i j}}{\partial V_{j}}=Y_{i j} V_{i},\left.\frac{\partial P_{i}}{\partial V_{k}}\right|_{k \neq i, k \neq j}=0
\end{gathered}
$$

In an MTDC system with large power flows and long transmission distances, the voltages might be significantly different from each other. From the system planning point of view, it might be more reasonable to specify the mean voltage of all the buses instead of that of the slack bus. A new problem can be presented as how to solve the power flow with the given powers and the mean voltage. An algorithm has been developed from the former power flow method to solve this problem.

Under this circumstance, all the $n$ voltages are configured to be the variables and an additional equation is built to represent the average voltage. The supplementary parametric equations and the associated Jacobian element are

$$
V_{a v}(\mathbf{V})=\frac{1}{n} \sum_{i=1}^{n} V_{i}, \quad \frac{\partial V_{a v}}{\partial V_{i}}=\frac{\partial}{\partial V_{i}}\left(\frac{1}{n} \sum_{i=1}^{n} V_{i}\right)=\frac{1}{n}
$$

where $V_{a v}$ is the specified average voltage of the grid. The other Jacobian elements can be computed according to (11) and (12), as the power flow equations remain as (9) and (10).

If the power injections are specified for all terminals except for the terminal $m$, this bus can be seen as a 'floating slack bus' aiming to achieve the given mean voltage. This power flow does not stand for any dc grid control strategy but could be very useful for system planning and converter reference setting.

From the control perspective, the two methods discussed in this section can be employed to configure the nominal voltage and power/current references for the droop line set.

\section{POWER Flow OF MTDC WITH DROOP CONTROL}

Voltage-power (V-P) and voltage-current (V-I) characteristics are the two most widely proposed droop control approaches. From a local converter control point of view, their behaviours are very similar to each other especially when the dc voltage is controlled close to its rated value. To compare the steady-state performance of these two types of droop, accurate power flow calculation methods are required.

\section{A. Voltage Power (V-P) Droop}

Frequency-power droop is used by synchronous generators in conventional ac systems to achieve a distributed frequency control. Based on the experience of ac grids, voltage-power droop is a strong candidate to be adopted in future MTDC grids. However, there is no equivalent 'AGC' type of control in MTDC systems to restore the dc voltage to its nominal value thus the V-P droop needs to be incorporated in power flows.

Generally, if V-P droop is employed by a dc grid of $n$ buses, the power flow problem can be described as how to solve the operating point of the system with a series of $m$ specified V-P characteristics and $(n-m)$ given nodal or branch powers.

If the $\mathrm{V}-\mathrm{P}$ droop is used for terminal $i$, the converter rectifying power would be controlled according to:

$$
P_{i}=K_{i}\left(V_{i}^{*}-V_{i}\right)+P_{i}^{*}
$$

where the voltage and power references of the droop line are denoted by $V_{i}^{*}$ and $P_{i}^{*}$. The droop control gain, which indicates the sensitivity of the converter power to the local dc voltage, is represented by $K_{i}$. Note that rectifier orientation is used through this paper for the direction of dc side power and current.

By setting $K$ to zero, a VSC terminal in power control mode or with known power generation can also be represented by equation (14). This feature of V-P droop makes it easier to analyse the power flow of the grid in a more generic way.

Voltages of all dc buses are selected as the variables to be solved. The vector of the specified parameters is comprised of the power references of the converters in droop control and the given power profile related to other converters:

$$
\mathbf{P}_{s p}=\left[\begin{array}{llll}
P_{1}^{*} & P_{2}^{*} & \cdots & P_{n}^{*}
\end{array}\right]^{T} .
$$

The non-linear functions related to the given nodal and branch powers are shown in (9) and (10). By combining (9) and the V-P characteristic equation (14), the parametric functions related to the droop are computed as:

$$
f_{i}(\mathbf{V})=V_{i}^{2} Y_{i i}+V_{i} \sum_{j=1, j \neq i}^{n} Y_{i j} V_{j}-K_{i} V_{i}^{*}+K_{i} V_{i}=P_{i}^{*} .
$$

The corresponding Jacobian elements can thus be obtained:

$$
\frac{\partial P_{i}}{\partial V_{i}}=2 Y_{i i} V_{i}+\sum_{j=1, j \neq i}^{n} Y_{i j} V_{j}+K_{i},\left.\frac{\partial P_{i}}{\partial V_{j}}\right|_{j \neq i}=V_{i} Y_{i j} .
$$

The offline converters can be considered as a specific V-P droop with both the gain $K$ and the power reference $P^{*}$ equal zero. If there are no branch power flows specified, all the non-linear equations and the Jacobian elements can be written in the form of (16) and (17), respectively. 


\section{B. Voltage Current (V-I) Droop}

The rectifying power of terminal $i$ equipped with a typical V-I droop can be represented as:

$$
P_{i}=V_{i} \cdot\left[K_{i}\left(V_{i}^{*}-V_{i}\right)+I_{i}^{*}\right]
$$

where the current reference is represented by $I_{i}^{*}$. Considering that the power $P_{i}$ can also be derived as (9) based on (8), the following objective function is obtained for terminals in V-I droop mode as

$$
f_{i}(\mathbf{V})=\left(Y_{i i}+K_{i}\right) V_{i}^{2}+V_{i}\left(\sum_{j=1, j \neq i}^{n} Y_{i j} V_{j}-\left(I_{i}^{*}+K_{i} V_{i}^{*}\right)\right)=0 .
$$

Accordingly, the specified parameters are obtained as:

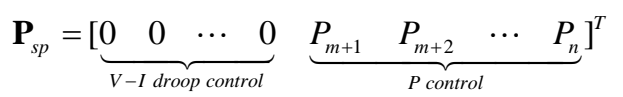

where zero is chosen to indicate the effectiveness of the droop control while the other $(\mathrm{n}-\mathrm{m})$ parameters are comprised of the specified nodal or line powers.

The Jacobian elements associated with (19) are derived as

$$
\begin{gathered}
\frac{\partial f_{i}}{\partial V_{i}}=2\left(Y_{i i}+K_{i}\right) V_{i}+\sum_{j=1, j \neq i}^{n} Y_{i j} V_{j}-\left(I_{i}^{*}+K_{i} V_{i}^{*}\right) \\
\left.\frac{\partial f_{i}}{\partial V_{j}}\right|_{j \neq i}=V_{i} Y_{i j} .
\end{gathered}
$$

So far, the steady-state equations for converters in slack bus control, constant power control, basic V-P and V-I droop control, and off-line operations have been addressed. Therefore, generalized MTDC power flow can be solved for dc grids with a mixture of these control strategies by appropriately integrating these equations using the NR method.

\section{POWER Flow WITH GENERALIZEd V-I/V-P CHARACTERISTICS}

In the algorithms discussed in Section IV and V, the operating mode of a converter is fixed and the converter limits have not been considered in detail. Furthermore, the droop control used in Section V has not been sufficiently generic. Realistically, the droop characteristics could be a combination of multiple linear or non-linear functions of dc voltage, such as the two types of droop lines illustrated in Fig. 6.

In a practical dc grid, a degree of voltage control capability could be required for all the VSCs. When the voltage droop with a power dead-band shown in Fig. 6(a) is implemented, the scheduled power will be produced by the converter as the dc voltage is maintained close to its nominal value. Once the voltage exceeds the dead-band zone, the converter power will adjust to contribute to the stabilization of the dc grid.

Shown in Fig. 6(b), voltage limits can be enabled by introducing two more stages of droop. As the overvoltage or undervoltage threshold is violated, the droop control gain $K$ will be increased in order to maintain the voltage within an acceptable range. The increased gain $K_{\max }$ should be kept within the dynamic requirement of the stability margin.

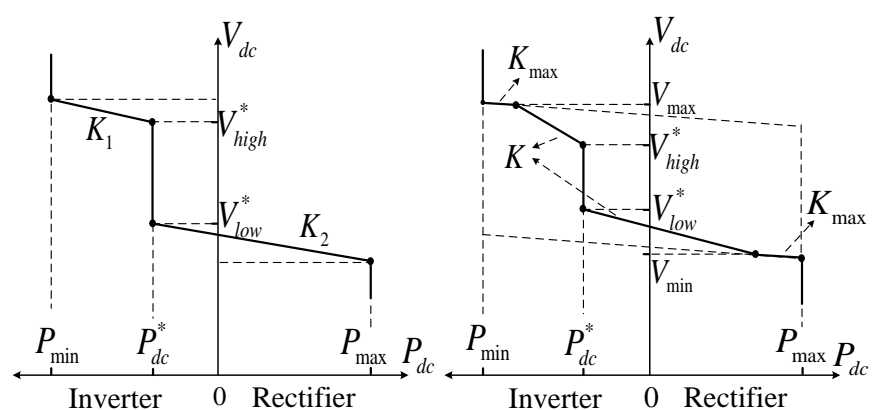

(a)

(b)

Fig. 6. (a) V-P droop with power dead-band; (b) V - P droop with dead-band and voltage limits.

An algorithm is proposed here to solve the MTDC power flow involving more complex droop characteristics with multiple control modes. This generalized approach can be applied to most types of VSC power and dc voltage control. Converter limit checking is included by enabling an additional outer iteration loop. The key procedure of this method is to iteratively update the parameters of V-P or V-I functions according to the newly estimated voltages.

Each voltage droop line is essentially a function between the voltage and desired current/power output. For example, the voltage droop with a dead-band and voltage limits is comprised of multiple linear functions. Differentiated by the voltage level, these linear functions of the converter power $P_{d c}\left(V_{d c}\right)$ can all be represented in a form of the typical droop lines:

$$
\begin{array}{lr}
K_{\max }\left(V_{\max }-V_{d c}\right)+\left[K\left(V_{h i g h}^{*}-V_{\max }\right)+P_{d c}^{*}\right], & \text { for } V_{d c} \geq V_{\max } \\
K\left(V_{\text {high }}^{*}-V_{d c}\right)+P_{d c}^{*}, & \text { for } V_{\text {high }}^{*}<V_{d c}<V_{\max } \\
0 \cdot\left(V_{\text {high }}^{*}-V_{d c}\right)+P_{d c}^{*}, & \text { for } V_{\text {low }}^{*} \leq V_{d c} \leq V_{\text {high }}^{*} \\
K\left(V_{\text {low }}^{*}-V_{d c}\right)+P_{d c}^{*}, & \text { for } V_{\min }^{*}<V_{d c}<V_{\text {low }} \\
K_{\max }\left(V_{\min }-V_{d c}\right)+\left[K\left(V_{\text {low }}^{*}-V_{\min }\right)+P_{d c}^{*}\right], \text { for } V_{d c} \leq V_{\min } .
\end{array}
$$

In fact, like the constant power control of an effective $K$ of zero, the slack bus control can be modelled as another extreme case of droop:

$$
P\left(V_{d c}\right)=K\left(V^{*}-V_{d c}\right), \quad K \rightarrow \infty .
$$

With the gain of the slack bus approximated by a sufficiently large number, a good accuracy can be achieved. Based on this, the voltage margin characteristic can be considered as a specific case of the voltage droop with a power dead-band. Basically, all the linear stages of different voltage control methods can be represented in the form of the droop function. This will significantly facilitate the power flow programming.

More generally, if the steady-state V-I function $I_{i}\left(V_{i}\right)$ or V-P function $P_{i}\left(V_{i}\right)$ is non-linear at some stages, the power flow equation representing the related terminal $i$ can be written as:

$$
\begin{gathered}
f_{i}=V_{i} \cdot I_{i}\left(V_{i}\right)-V_{i} \cdot \sum_{j=1}^{n} Y_{i j} V_{j}=0 \\
f_{i}=P_{i}\left(V_{i}\right)-V_{i} \cdot \sum_{j=1}^{n} Y_{i j} V_{j}=0
\end{gathered}
$$




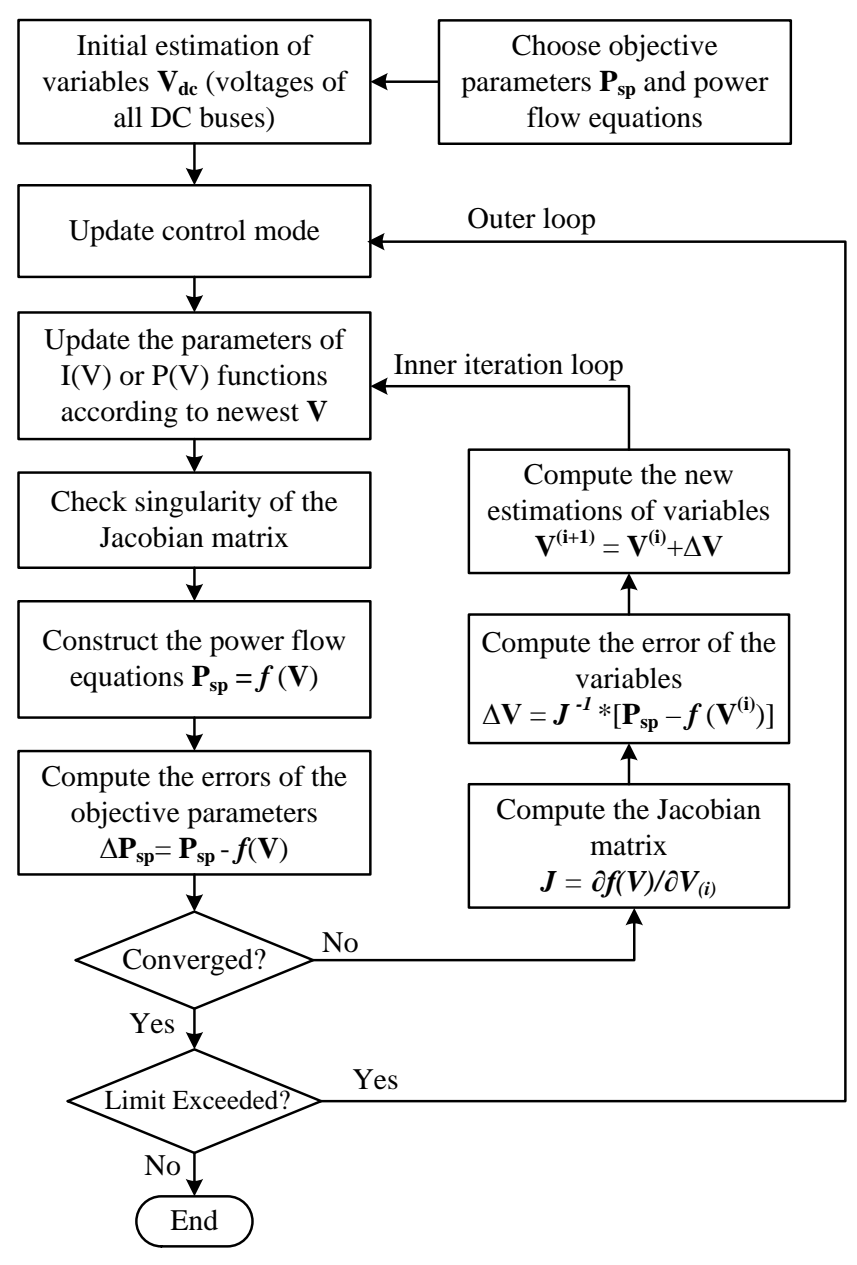

Fig. 7. Flow chart of the dc power flow with generic droop lines.

In the $k$ th iteration, using the estimated voltages $\mathbf{V}^{(k)}$, the associated Jacobian elements can be derived as (27) and (28).

$$
\begin{gathered}
\frac{\partial f_{i}}{\partial V_{i}^{(k)}}=I_{i}\left(V_{i}^{(k)}\right)+V_{i}^{(k)} \cdot \frac{d I_{i}\left(V_{i}^{(k)}\right)}{d V_{i}^{(k)}}-2 Y_{i i} V_{i}^{(k)}-\sum_{j=1, j \neq i}^{n} Y_{i j} V_{j}^{(k)} \\
\frac{\partial f_{i}}{\partial V_{i}^{(k)}}=\frac{d P_{i}\left(V_{i}^{(k)}\right)}{d V_{i}^{(k)}}-2 Y_{i i} V_{i}^{(k)}-\sum_{j=1, j \neq i}^{n} Y_{i j} V_{j}^{(k)}
\end{gathered}
$$

With the equations derived for each control scheme, the generalized algorithm can therefore be utilized to integrate them together, with the corresponding flow chart illustrated in Fig. 7. The algorithm consists of an outer iteration loop to check converter limits and an inner iteration loop to perform NR calculations. Two important features of the inner loop are to select the correct V-I and V-P functions and to ensure a non-singular Jacobian matrix.

The control modes and the corresponding parameters of the $\boldsymbol{I}(\mathbf{V})$ and $\boldsymbol{P}(\mathbf{V})$ functions will be kept up-to-date in each iteration according to the newest set of voltage estimates. Specifically, if the voltage characteristics are linear, the dc voltages act as an indication of the values of the gain $K$ and the references $\left(V^{*}, P^{*}, I^{*}\right)$ which need to be used for the next iteration. If the converter limit mode is activated by the outer iteration loop, the parameters need to be fixed to the limit throughout the inner NR iteration loop.

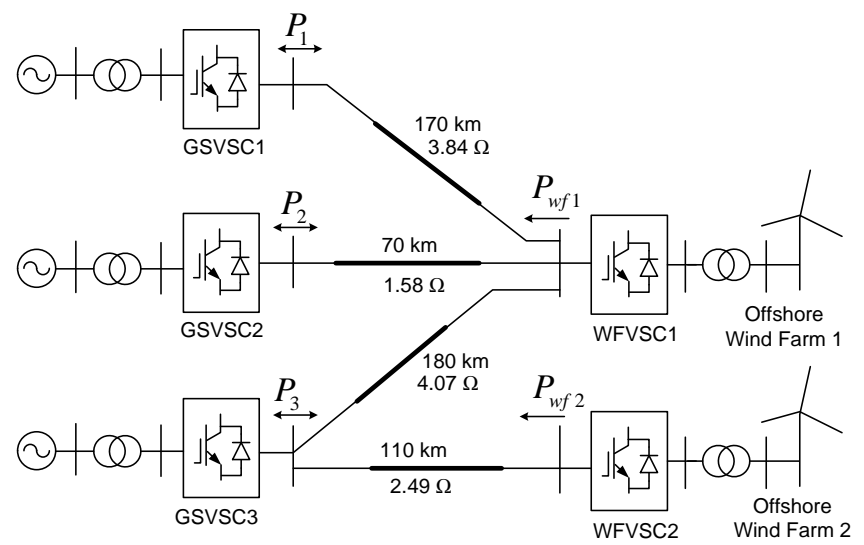

Fig. 8. Five-terminal test network for power flow studies.

Under certain situations where the voltage estimates indicate that all the terminals ought to be in dead-band mode or constant power/current mode, the corresponding Jacobian will not be invertible as the voltages will effectively become unsolvable. In this case, a temporary approximation is used to modify the effective $K$ of the dead-band control mode from zero to a small value such as 0.5 . This will therefore avoid the singularity of the Jacobian and allow the iteration to continue. When the voltage estimates correspond to a system state where at least one converter is in voltage control mode $(K \neq 0)$, the gains for all the dead-band modes will be set back to zero. This problem will not occur if no dead-band is involved in the droop lines. The approximation will not affect the final power flow results.

After the convergence of the inner iteration, the resulting converter powers or currents will be examined, as it is noted that the converter limits are not considered in the inner iteration loop. If the limits are exceeded, the related terminals will be switched to constant power or constant current mode and the calculation enters another series of inner NR iterations. Either dc power or dc current limit or a combination of both can be implemented. It may be more reasonable to employ a power limit under an over-voltage condition, while a current limit could be required under an under-voltage circumstance.

\section{CASE STUdies AND SimUlations}

This section demonstrates the performances of the proposed power flow methods and evaluates the quasi-steady-state behaviours of various dc voltage control techniques after transient events including wind power changes and converter outages. The power flow algorithms and the case studies are implemented in MATLAB.

\section{A. Test System and Sets of Droop Lines}

A five-terminal VSC-HVDC network, shown in Fig. 8, is established as the candidate dc grid model. All the converter stations are rated at $1000 \mathrm{MW}, \pm 320 \mathrm{kV}$, with symmetrical monopole topology. This model is configured to enable a primary power flow from the grid side converter station 1 (GSVSC1) to GSVSC2 and GSVSC3, with the integration of two offshore wind farms. The aggregated resistance of the positive-pole and negative-pole submarine cables is provided.

Presented in Fig. 9, the five control scenarios comprising various combinations of voltage characteristics, are designed 


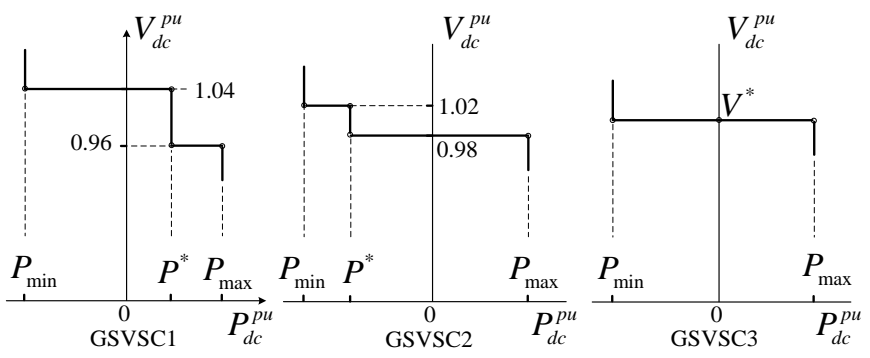

(a) Case 1: voltage margin characteristics

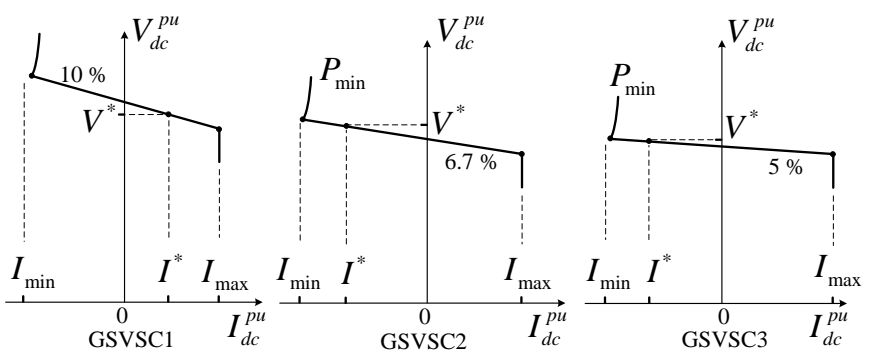

(b) Case 2: voltage-current droop

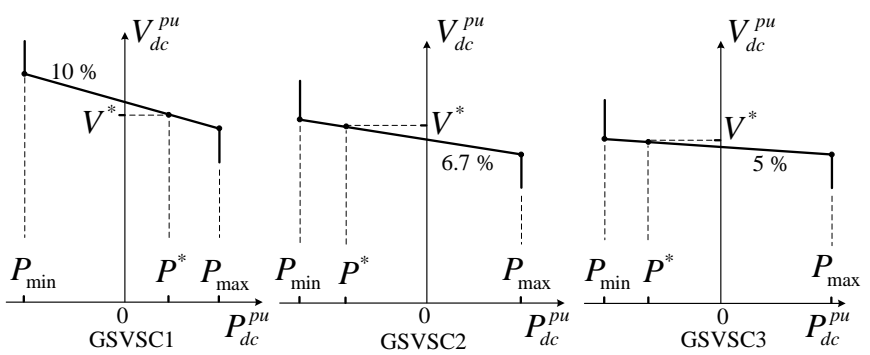

(c) Case 3: voltage-power droop

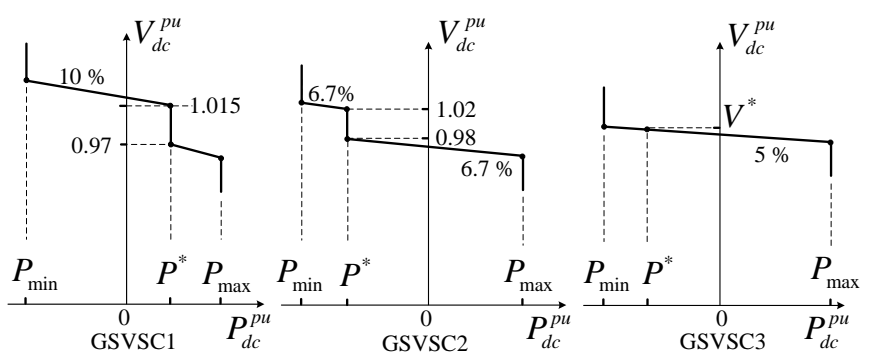

(d) Case 4: V-P droop with power dead-band

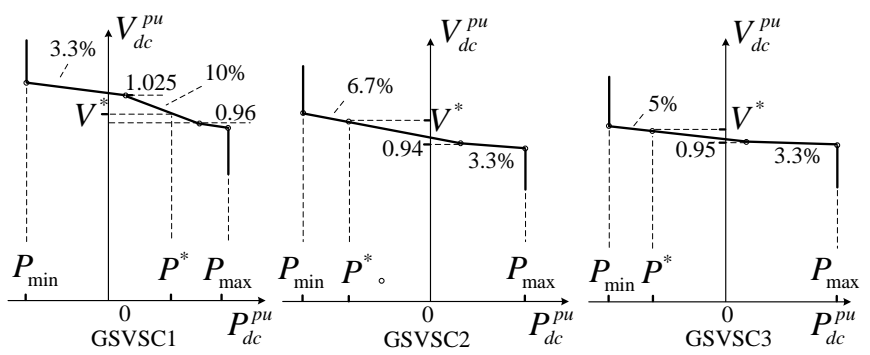

(e) Case 5: V-P droop with voltage limits

Fig. 9. DC voltage characteristics of the three grid side converters.

for the three GSVSCs. All the parameters are shown in per unit. The droop constant $\rho$, which equals $1 / K$, is provided for each droop stage. The highest priority of controlling the dc voltage is allocated to GSVSC3, and GSVSC1 is scheduled with the
TABLE I

Power Flow Results of The Nominal Operating Point

\begin{tabular}{cccccc}
\hline Converter & GSVSC1 & GSVSC2 & GSVSC3 & WFVSC1 & WFVSC2 \\
\hline$V_{d c}$ (p.u.) & 0.9999 & 0.9921 & 0.9923 & 0.9953 & 0.9954 \\
$P_{d c}$ (p.u.) & 0.5000 & -0.8000 & -0.7927 & 0.6000 & 0.5000 \\
$I_{d c}$ (p.u.) & 0.5000 & -0.8063 & -0.7989 & 0.6029 & 0.5023 \\
\hline
\end{tabular}

lowest priority, which can be observed from the configurations of the droop gains and the dead-band/margin ranges. DC power and current limits of 1.05 pu are employed in the five scenarios. Key factors involving the allowable dc voltage variations, the power sharing between converters, the participation on stabilising the grid, and under/over dc voltage control, are considered in configuring the droop characteristics.

The nominal operating point of the system, shown in Table I, is obtained via the method shown in Section IV, with specified powers for all the converters except GSVSC3 and an average dc voltage of $0.995 \mathrm{pu}$. These power flow results are employed as the references $\left(V^{*}, P^{*}, I^{*}\right)$ for all the five sets of droop lines. The voltage margin between the GSVSCs in Case 1 is configured to be relatively large in order to avoid unwanted control interactions. For each VSC, identical droop constants are utilized in the V-I droop in Case 2 and the V-P droop in Case 3. In case of power imbalance, the largest power deviation and the lowest voltage deviation are expected to be experienced by GSVSC3, which has the smallest droop constant. In Case 2, the maximum power is limited instead of maximum current under inverter operation. Unlike the margin control, the dead-bands for GSVSC1 and GSVSC2 in Case 4 are arranged to be closed to each other as there is no necessity to avoid the interactions between droop lines. The voltage limits in Case 5 allow a tighter control of the post-transient voltage.

The generalized procedure proposed in Section VI is employed in all the scenarios, integrating the power flow equations listed in Section IV-VI. An approximated droop gain of $10^{8}$ is used for the voltage margin control. It has been tested that the errors due to this approximation are within $10^{-8}$ for $\mathrm{dc}$ voltages and are less than $10^{-10}$ for powers.

\section{B. Wind Power Variations}

A series of power flows are solved with the five control cases implemented, as the rectifying power of WFVSC2 varies from 0 to 1.0 pu while the power injection of WFVSC1 fixed at 0.9 $\mathrm{pu}$. The steady-state responses of the converter powers for Case 1, 2, 4, 5 are presented in Fig. 10. As the voltages of all the buses are close to each other, only the average voltage is shown here. In addition, with the error tolerance of $10^{-8}$ applied, the iteration numbers required by each control case are also presented as functions of the power of WFVSC2 in Fig. 11.

It is clearly demonstrated in Fig. 10 that for the voltage margin control there is only one converter at a time responding to dc voltage variation. The average voltage increases slightly even without change of the slack bus, in that the WFVSC2 bus voltage rises as more power is injected to the grid.

The results for Case 2 and 5 demonstrate that the differences between the behaviours of the V-I and V-P droop lines are almost negligible until the voltage limit control of Case 5 is activated. Unlike the margin control in Case 1, the steady-state voltage response with droop control is continuous. The voltage 

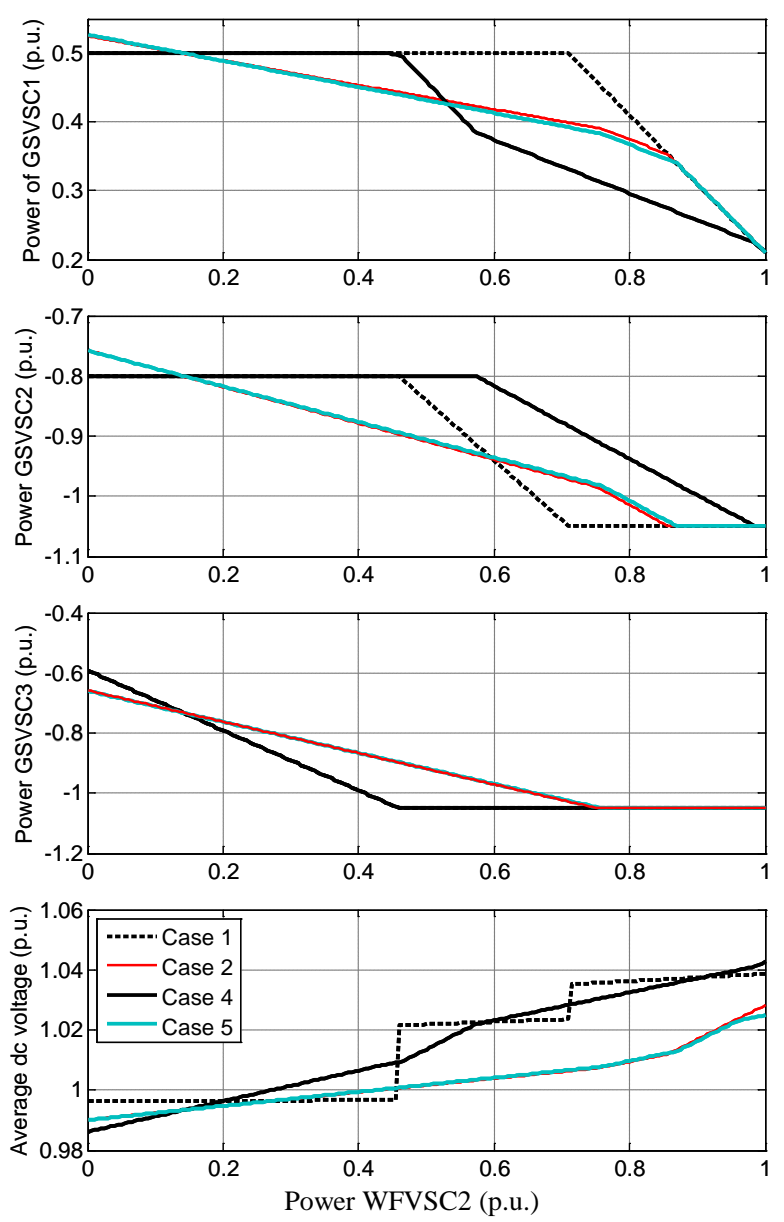

Fig. 10. Steady-state variations of the power generations of the GSVSCs and the average dc voltage.

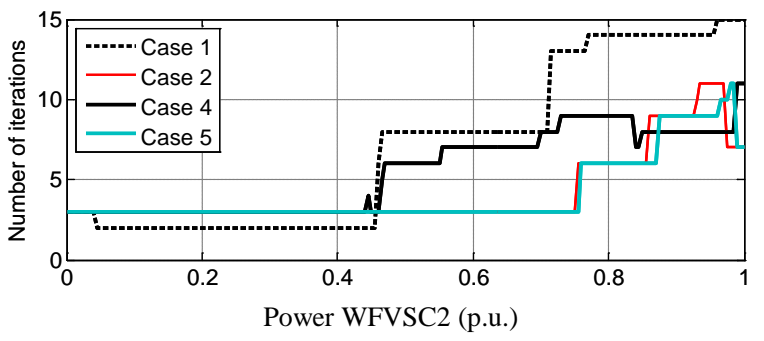

Fig. 11. Number of iterations for the five control cases for a series of wind power generations.

rises with the increase of the wind generation due the negative-feedback nature of the droop (proportional) control. For a fixed amount of the wind power variations, as more VSCs enter their limit modes, both the power and voltage deviations of the remaining VSCs in droop mode will increase. A smaller droop constant implies that the power of the associated terminal responds more abruptly to the wind power changes.

Shown by the power flow solutions of Case 4, the powers of both GSVSC1 and GSVSC2 are not perturbed in the dead-band range until the droop slack bus reaches its limit. The transitions of the power sharing role are clearly indicated, and that the priorities in response to the power disturbances are determined by the configuration of the dead-band ranges is demonstrated.
TABLE II

POWER Flow Results OF THE OUTAGE OF GSVSC1 (IN P.U.)

\begin{tabular}{c|c|ccccc}
\hline \multicolumn{2}{c|}{ Converters } & Case 1 & Case 2 & Case 3 & Case 4 & Case 5 \\
\hline \multirow{2}{*}{ GSVSC2 } & $V_{d c}$ & 0.9871 & 0.9773 & 0.9769 & 0.9710 & 0.9769 \\
& $P_{d c}$ & -0.8000 & -0.5704 & -0.5720 & -0.6644 & -0.5720 \\
\hline \multirow{2}{*}{ GSVSC3 } & $V_{d c}$ & 0.9923 & 0.9793 & 0.9789 & 0.9743 & 0.9789 \\
& $P_{d c}$ & -0.2955 & -0.5267 & -0.5251 & -0.4322 & -0.5251 \\
\hline
\end{tabular}

TABLE III

POWER Flow RESUlts OF THE OUTAGE OF GSVSC2 (IN P.U.)

\begin{tabular}{c|c|ccccc}
\hline \multicolumn{2}{c|}{ Converters } & Case 1 & Case 2 & Case 3 & Case 4 & Case 5 \\
\hline \multirow{2}{*}{ GSVSC1 } & $V_{d c}$ & 1.0400 & 1.0543 & 1.0545 & 1.0696 & 1.0382 \\
& $P_{d c}$ & -0.0458 & -0.0459 & -0.0459 & -0.0460 & -0.0457 \\
\hline \multirow{2}{*}{ GSVSC3 } & $V_{d c}$ & 1.0351 & 1.0495 & 1.0497 & 1.0649 & 1.0333 \\
& $P_{d c}$ & -1.0500 & -1.0500 & -1.0500 & -1.0500 & -1.0500 \\
\hline
\end{tabular}

TABLE IV

Power FlOW Results OF THE OUtAGE Of GSVSC3 (IN P.U.)

\begin{tabular}{c|c|ccccc}
\hline \multicolumn{2}{c|}{ Converters } & Case 1 & Case 2 & Case 3 & Case 4 & Case 5 \\
\hline \multirow{2}{*}{ GSVSC1 } & $V_{d c}$ & 1.0400 & 1.0540 & 1.0542 & 1.0693 & 1.0381 \\
& $P_{d c}$ & -0.0424 & -0.0426 & -0.0426 & -0.0428 & -0.0424 \\
\hline \multirow{2}{*}{ GSVSC2 } & $V_{d c}$ & 1.0365 & 1.0505 & 1.0507 & 1.0658 & 1.0345 \\
& $P_{d c}$ & -1.0500 & -1.0500 & -1.0500 & -1.0500 & -1.0500 \\
\hline
\end{tabular}

TABLE V

POWER Flow Results OF THE OUTAge OF WFVSC1 (IN P.U.)

\begin{tabular}{c|c|ccccc}
\hline \multicolumn{2}{c|}{ Converters } & Case 1 & Case 2 & Case 3 & Case 4 & Case 5 \\
\hline \multirow{2}{*}{ GSVSC1 } & $V_{d c}$ & 0.9940 & 0.9863 & 0.9863 & 0.9751 & 0.9863 \\
& $P_{d c}$ & 0.5000 & 0.6278 & 0.6364 & 0.5000 & 0.6364 \\
\hline \multirow{2}{*}{ GSVSC2 } & $V_{d c}$ & 0.9861 & 0.9780 & 0.9779 & 0.9678 & 0.9779 \\
& $P_{d c}$ & -0.8000 & -0.5815 & -0.5869 & -0.6172 & -0.5869 \\
\hline \multirow{2}{*}{ GSVSC3 } & $V_{d c}$ & 0.9923 & 0.9799 & 0.9798 & 0.9715 & 0.9798 \\
& $P_{d c}$ & -0.1926 & -0.5396 & -0.5426 & -0.3770 & -0.5426 \\
\hline
\end{tabular}

It is found in Fig. 11 that the total number of iterations required by the power flow computation is determined by the number of outer iterations. Generally, longer computation time is required for a system state with more converters in limit mode. If all the converters remain in normal operation mode, no more than 3 iterations are required for a strict tolerance of $10^{-8}$. In addition, more iterations are demanded in case of abrupt changes of control modes, such as the voltage margin control and the dead-band control.

The power flow method can be extended to involve wind farm control with some degree of fault ride-through capability by representing wind power using more realistic characteristics.

\section{Converter Outages}

The power flow method is employed here for the analysis of the impact of converter outages. It is assumed that the loss of the VSC is caused by the disconnection of the converter ac side. Four converter outage scenarios are studied. The resulting dc voltage and power profiles of the remaining GSVSCs are presented in Table II-V.

Generally, for an MTDC system based on droop control, loss of a rectifier will result in the drop of dc voltage and the increase of rectifying power (or reduction of inverting power) 


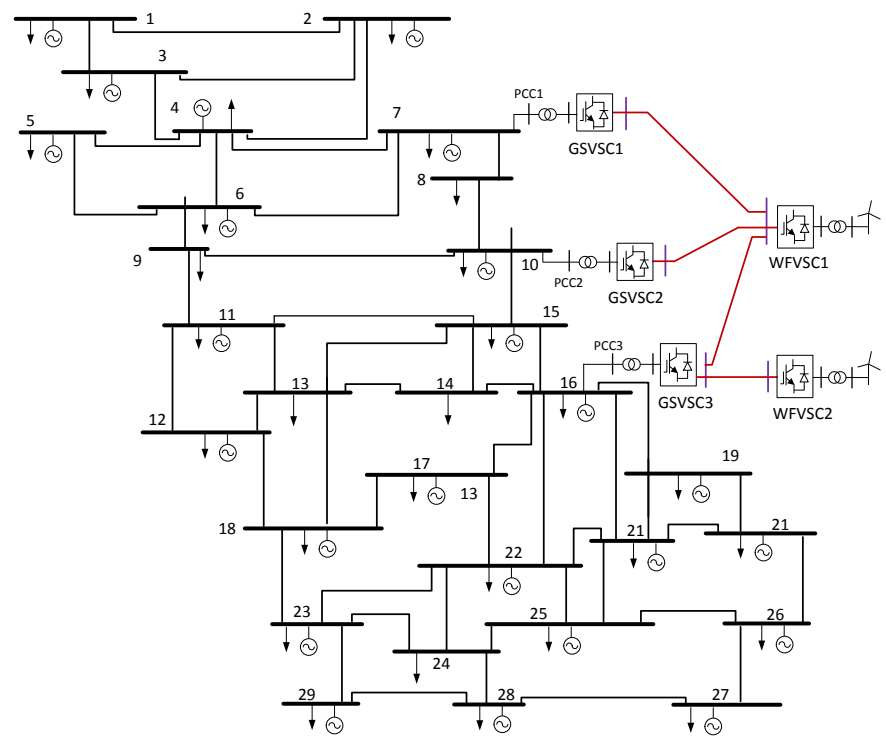

Fig. 12. Test system for integrated ac/dc power flow.

for the remaining terminals in droop control. Loss of an inverter will cause the rise of dc voltage and the decline of rectifying power of the VSCs in droop control. This can be observed from the results of Case 2, 3 and 5 in Table II-V.

After the outage of GSVSC1, GSVSC3 remains as the slack bus in the voltage margin case. Shown in TABLE II, the lowest voltages occur in Case 4 in that GSVSC2 does not contribute to maintaining the voltage until the dead-band is violated.

As GSVSC2 goes offline, as the only remaining inverter, GSVSC3 is not capable to absorb all the wind farm power and the voltage regulation role is taken over by GSVSC1. Power reversal is experienced by GSVSC1. The impact of the voltage limit control can be observed from the results of Case 5 in TABLE III. Similar results are obtained for the loss of GSVSC3. The new system state is dominated by the voltage characteristic of GSVSC1 as eventually it becomes the only terminal which has dc voltage control capability.

For Case 2, 3, 5, the power imbalance resulted from the loss of WFVSC1 is shared by the three onshore VSCs, according to the settings of droop constants. The dead-band control in Case 4 enables the powers of GSVSC2 and GSVSC1 to be less perturbed or even unchanged however this could imply a relatively large drift of the dc voltage.

\section{Case Study for AC/DC power flow}

In the previous test cases, the dc power flow methods have been evaluated across a range of control scenarios and disturbance conditions. The integrated ac/dc algorithm discussed in Section III has also been implemented on a 29-bus ac test network [21], with the single line diagram shown in Fig. 12. The converters GSVSC $1-3$ are connected to the $400 \mathrm{kV}$ ac buses 7, 10 and 16 respectively. The control case 4 in Fig. 9 is adopted for the GSVSCs in this case study. The power loss parameters derived based on the data from [22], and the parameters of the converter transformer and reactor, are shown in Table VI.

The combined ac/dc power flow is performed under weak and strong wind scenarios, with the results illustrated in
TABLE VI

CONVERTER AND POWER LOSS PARAMETERS

\begin{tabular}{cc|cc}
\hline \multicolumn{2}{c|}{ Converter station parameters } & \multicolumn{2}{c}{ Power loss data } \\
\hline$X_{\text {tr }}($ p.u. $)$ & 0.1400 & $K_{1}(k V)$ & 1.4932 \\
$R_{t r}$ (p.u. $)$ & 0.0050 & $K_{2}(k V)$ & 0.0802 \\
$X_{c}$ (p.u.) & 0.0750 & $K_{3}(\Omega)$ & 2.2080 \\
$R_{c}$ (p.u.) & 0.0025 & $K_{4}(\Omega)$ & 0.1227 \\
\hline
\end{tabular}

TABLE VII

POWER FLOW RESUltS FOR GSVSCs ( $\mathrm{P}_{\mathrm{WF} 1} 200$ MW, $\mathrm{P}_{\mathrm{WF} 2} 200 \mathrm{MW}$ )

\begin{tabular}{c|ccc}
\hline & GSVSC1 & GSVSC2 & GSVSC3 \\
\hline PCC bus type & $P V$ & $P Q$ & $P V$ \\
$P_{g}(M W)(I N V)$ & -504.679 & 601.593 & 303.650 \\
$Q_{g}(M V A r)$ & -33.540 & 100.000 & 102.840 \\
$V_{g}$ (p.u.) & 0.9980 & 1.0045 & 1.0050 \\
$\theta_{g}($ deg $)$ & 28.680 & 24.923 & 18.237 \\
$V_{d c}$ (p.u. $)$ & 0.9735 & 0.9663 & 0.9667 \\
$P_{d c}(M W)(R E C)$ & 500.000 & -595.232 & -300.462 \\
\hline Iteration time $(s)$ & $0.3475(\mathrm{ac})$ & & $0.0215(\mathrm{dc})$ \\
\hline
\end{tabular}

TABLE VIII

POWER FLOW RESUlts FOR GSVSCs ( $\mathrm{P}_{\mathrm{WF} 1} 950 \mathrm{MW}, \mathrm{P}_{\mathrm{WF} 1} 850 \mathrm{MW}$ )

\begin{tabular}{c|ccc}
\hline & GSVSC1 & GSVSC2 & GSVSC3 \\
\hline PCC bus type & $P Q$ & $P Q$ & $P Q$ \\
$P_{g}(M W)(I N V)$ & -259.718 & 1014.246 & 1067.270 \\
$Q_{g}(M V A r)$ & 0.000 & 0.000 & 0.000 \\
$V_{g}$ (p.u.) & 0.9999 & 1.0037 & 0.9986 \\
$\theta_{g}($ deg $)$ & 34.678 & 30.741 & 23.881 \\
$V_{d c}($ p.u. $)$ & 1.0393 & 1.0332 & 1.0350 \\
$P_{d c}(M W)(R E C)$ & 257.130 & -998.508 & -1050.000 \\
\hline Iteration time $(\mathrm{s})$ & $1.9639(\mathrm{ac})$ & & $0.0216(\mathrm{dc})$ \\
\hline
\end{tabular}

Table VII and Table VIII respectively. The ac system power flows are calculated by the open-source package MATPOWER [23]. The voltage magnitude $V_{g}$ and angle $\theta_{g}$ at the PCC bus, and the real and reactive power injection into the ac grid from the PCC bus, are illustrated. For the large wind generation scenario, the GSVSCs are changed from ac voltage control to reactive power control to ensure maximum power transfer. Due to the scale and the limited number of variables of the dc grid, the dc power flow requires much less computation time than the ac power flow iterations. Reduced calculation time is expected for the unified ac/dc power flow however that requires greater modifications and will reduce the flexibility of both ac and dc grid settings. The results demonstrate that the configuration of this set of droop lines enable a balance between supporting the north-south load flow of the onshore grid and the integration of offshore wind farms. It also suggests that the MTDC power flow is predominantly influenced by the configurations of the droop and the power sources of uncertainty (e.g. wind farms), with the ac power flow only imposing limited auxiliary impact.

\section{CONCLUSION}

A new generic power flow approach for MTDC systems with various types of dc voltage control has been presented in this paper. This method can be extended for detailed steady-state 
analysis of large dc grids with different network topologies and complex voltage characteristics.

For different MTDC control strategies, a non-linear power flow methodology and control equations have been identified. A power flow procedure based upon two layers of iteration loop is introduced to enable the calculation of voltage characteristics with voltage characteristics containing multiple control stages. The method to integrate this MTDC power flow with a conventional ac power flow has been proposed. This method utilizes the fact that for dc V-I/V-P droop control of VSC-HVDC the power flows within the dc system can be solved separately from ac power flows.

The proposed procedure has been applied to a series of test cases. In the case studies, several sets of voltage characteristics are implemented for the GSVSCs in the five-terminal system. The combined ac/dc power flow algorithm has been tested on a meshed ac network. The quasi-steady-state responses of the system to a range of wind power disturbances and converter outages are presented. The post-disturbance behaviours of different control scenarios have been compared. The power flow method has demonstrated its performance in obtaining the results of the case studies.

\section{ACKNOWLEDGEMENT}

The authors would like to thank L. Shen, K. R. W Bell, and M. Belivanis for their input on ac system modelling.

\section{REFERENCES}

National Grid, Offshore Development Information Statement 2011, Sep 2011. [Online]. Available: http://www. nationalgrid.com/uk/Electricity/OffshoreTransmission/

[2] ABB, HVDC reference projects in Europe, [Online]. Available: http://www.abb.com

[3] China EPRI, China's Electric Power-Our Responsibility, [Online]. Available: http://www.epri.sgcc.com.cn/prgc/

[4] M. Barnes and A. Beddard, "Voltage Source Converter HVDC Links - The State of the Art and Issues Going Forward," Energy Procedia, vol. 24, pp. 108-122, 2012.

[5] T. Nakajima and S. Irokawa, "A control system for HVDC transmission by voltage sourced converters," in Proc. IEEE PES Summer Meeting, Jul. 1999, pp. 1113-1119. .

[6] C. Dierckxsens, K. Srivastava, M. Reza, S. Cole, J. Beerten, and R. Belmans, "A distributed DC voltage control method for VSC MTDC systems," Elect. Power Syst. Res., vol. 82, pp. 54-58, 2012.

[7] T. M. Haileselassie and K. Uhlen, "Impact of DC Line Voltage Drops on Power Flow of MTDC Using Droop Control," IEEE Trans. Power Syst., vol. 27, pp. 1441 - 1449, 2012.

[8] O. Gomis-Bellmunt, J. Liang, J. Ekanayake, and N. Jenkins, "Voltage-current characteristics of multiterminal HVDC-VSC for offshore wind farms," Elect. Power Syst. Res., vol. 81, pp. 440-450, 2011.

[9] CIGRE WG B4-52, "HVDC Grid feasibility study," CIGRE Brochure 533, Apr. 2013.

[10] X. Zhang, "Multiterminal voltage-sourced converter-based HVDC models for power flow analysis," IEEE Trans. Power Syst., vol. 19, pp. 1877-1884, 2004.

[11] J. Beerten, S. Cole, and R. Belmans, "Generalized Steady-State VSC MTDC Model for Sequential AC/DC Power Flow Algorithms," IEEE Trans. Power Syst., vol. 27, pp. 821-829, 2012.

[12] T. M. Haileselassie and K. Uhlen, "Power flow analysis of multi-terminal HVDC networks," in Proc. PowerTech, Trondheim, Norway, 2011, p. 6.

[13] M. Aragüés-Peñalba, A. Egea-Àlvarez, O. Gomis-Bellmunt, and A. Sumper, "Optimum voltage control for loss minimization in HVDC multi-terminal transmission systems for large offshore wind farms," Elect. Power Syst. Res., vol. 89, pp. 54-63, 2012.
[14] R. Teixeira Pinto, P. Bauer, S. Rodrigues, E. Wiggelinkhuizen, J. Pierik, and J. Ferreira, "A novel distributed direct voltage control strategy for grid integration of offshore wind energy systems through MTDC network," IEEE Trans. Ind. Electron., vol. 60, pp. 2429 - 2441, 2012.

[15] J. Beerten, D. Van Hertem, and R. Belmans, "VSC MTDC systems with a distributed DC voltage control - A power flow approach," in Proc. IEEE PowerTech, Trondheim, Norway, 2011, p. 6.

[16] C. Oates and C. Davidson, "A comparison of two methods of estimating losses in the Modular Multi-Level Converter," in Proc. 14th European Conf. on Power Electron. and Applicat., 2011, pp. $1-10$.

[17] IEC SC 22F, "Determination of power losses in voltage sourced converters (VSC) for HVDC systems: part 1 - general requirements," IEC 62751-1, 2012.

[18] A. Petersson, "Analysis, Modeling and Control of Doubly-Fed Induction Generators for Wind Turbines," Ph.D. dissertation, Chalmers Univ. of Technol., Gothenburg, Sweden, 2003.

[19] W. F. Tinney and C. E. Hart, "Power Flow Solution by Newton's Method," IEEE Trans. Power App. Syst., vol. PAS-86, pp. 1449-1460, 1967.

[20] A. J. Wood and B. F. Wollenberg, Power generation, operation and control, 2nd ed. New York: Wiley, 1996.

[21] K. R. W. Bell and A. N. D. Tleis, "Test system requirements for modelling future power systems," in Proc. Power and Energy Society General Meeting, 2010 IEEE, 2010, pp. 1-8.

[22] ABB, ABB HiPak IGBT Module 5SNA 1200G450350, 2012. [Online]. Available: www.abb.com/semiconductors

[23] R. D. Zimmerman, C. E. Murillo-Sanchez, and R. J. Thomas, "MATPOWER: Steady-State Operations, Planning, and Analysis Tools for Power Systems Research and Education," IEEE Trans. Power Syst., vol. 26, pp. 12-19, 2011.

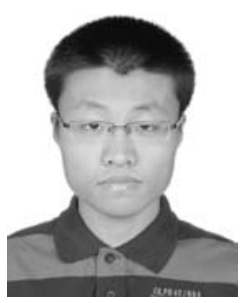

Wenyuan Wang (S'12) received the B.Eng. degree in electrical and electronic engineering in 2011, from The University of Manchester, Manchester, U.K., where he is currently pursuing the Ph.D. degree. His research interests include operation, stability and control of multi-terminal VSC-HVDC systems.

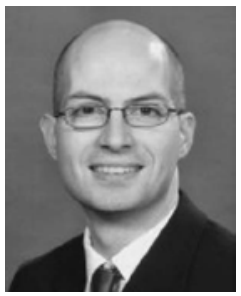

Mike Barnes (M'96-SM'07) received the B.Eng. and Ph.D. degrees from the University of Warwick, Coventry, U.K. In 1997, he was a Lecturer with the University of Manchester Institute of Science and Technology (UMIST, now merged with The University of Manchester), Manchester, U.K., where he is currently a Professor. His research interests cover the field of power-electronics-enabled power systems. 